Cienc Tecn UTEQ (2017) 10(2) p 29-36 ISSN 1390-4051; e-ISSN 1390-4043 (cc) (i) DOI: https://doi.org/10.18779/cyt.v11i1.187

\title{
Análisis de tres elementos de aforo para sistemas de riego en la provincia de Loja, Ecuador
}

\author{
Analysis between three gauging elements for irrigation systems in the province of Loja, Ecuador \\ María Narcisa Chamba Ontaneda ${ }^{1}$,Holger Benavides-Muñoz ${ }^{1}$ \\ ${ }^{1}$ Universidad Técnica Particular de Loja (UTPL), Departamento de geología, minas e ingeniería civil, \\ Maestríade recursos hídricos.merys15@gmail.com; hmbenavides@utpl.edu.ec
}

Rec.: 27.02.2018. Acept.: 04.06.2018. Publicado el 2 de julio de 2018

\section{Resumen}

$E_{d e}^{1}$ lobjetivo del estudio fue analizar los tres elementos típicos

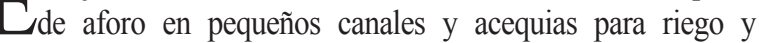
generar modelos matemáticos que gobiernan su comportamiento hidrodinámico. Los dispositivos que se analizaron fueron la canaleta Parshall (CP), el aforador con cuello (AC) y el aforador sin cuello (AS). La investigación se desarrolló en tres fases. En las primeras etapas se determinó las descargas entre $0 \mathrm{~L} / \mathrm{s}-10$ $\mathrm{L} / \mathrm{s}$, el número de mediciones fue de nueve por cada elemento, con un total de 54 medidas y finalmente en el banco hidráulico del laboratorio se generaron las ecuaciones de calibración y curvas características de los medidores de caudal. Los resultados obtenidos muestran una variación entre $0.001 \mathrm{~L} / \mathrm{s}$ a $0.002 \mathrm{~L} / \mathrm{s}$ para descargas menores a $2 \mathrm{~L} / \mathrm{s}$; y, de $0.008 \mathrm{~L} / \mathrm{s}$ a $0.063 \mathrm{~L} / \mathrm{s}$ para gastos entre $2 \mathrm{~L} / \mathrm{s}$ y $10 \mathrm{~L} / \mathrm{s}$. Se establecieron las ecuaciones y curvas de calibración para la determinación del caudal en L/s en función de la altura del tirante en mm. La simulación del flujo en los aforadores obedeció a un comportamiento del flujo bajo régimen supercrítico.

Palabras clave: aforo, riego, dispositivos de aforo, canaletas, canaleta Parshall.

\begin{abstract}
$\mathrm{T}$ The objective of the study was to analyze the three typical elements of gauging in small channels and ditches for irrigation and to generate mathematical models that govern their hydrodynamic behavior. The devices analyzed were the Parshall gutter (CP), the neck gauge (AC) and the neckless gauge (AS). The research was developed in three phases. In the first stages, the discharges between $0 \mathrm{~L} / \mathrm{s}$ and $10 \mathrm{~L} / \mathrm{s}$ were determined, the number of measurements was nine for each element, with a total of 54 measurements, and finally the calibration equations, and the curves characterizing the flow meters were generated in the hydraulic bench of the laboratory. Results show a variation between $0.001 \mathrm{~L} / \mathrm{s}$ and $0.002 \mathrm{~L} / \mathrm{s}$ for discharges of less than $2 \mathrm{~L} / \mathrm{s}$; and from $0.008 \mathrm{~L} / \mathrm{s}$ to 0.063 $\mathrm{L} / \mathrm{s}$ for expenses between $2 \mathrm{~L} / \mathrm{s}$ and $10 \mathrm{~L} / \mathrm{s}$. The equations and calibration curves were established to determine the flow rate in $\mathrm{L} / \mathrm{s}$ as a function of the height of the tie rod in $\mathrm{mm}$. The simulation of flow in the gauges was due to flow behavior under supercritical regime.
\end{abstract}

Keywords: gauging, irrigation, gauging devices, gutter. Parshall gutter. 


\section{Introducción}

$\mathrm{L}$ as crecientes dificultades para acceder al recurso hídrico se debe a múltiples factores, entre los que se entrevé la expansión de la frontera agrícola, que tiene implícito el aumento progresivo de la superficie tanto para cultivo como para riego, sumado a un apremiante incremento poblacional, que hace que la demanda hídrica se amplifique para satisfacer todas sus necesidades vitales.

La gran mayoría de sistemas de abastecimiento de agua para riego en Ecuador aún no brindan las facilidades técnicas para practicar un efectivo aforo de caudales, aspecto primordial para una distribución con equidad, a la vez, es la base de la tan anhelada gestión sostenible del recurso.

Loja es una provincia eminentemente agrícola, su economía se basa en la producción de alimentos tales como legumbres, verduras, frutas y hortalizas. La agricultura está asociada directamente al clima y a los procesos naturales (Riera y Pereira, 2012). En la época seca se evidencia la disminución de las precipitaciones y del caudal disponible en las cabeceras de las parcelas, por lo que se requiere realizar una correcta medición y distribución del agua disponible, según lo establezcan las modulaciones de riego y las necesidades del cultivo.

En el estudio se analizaron los aforadores más comunes como la canaleta Parshall (CP), el aforador con cuello (AC) y el aforador sin cuello (AS). El primero de estos instrumentos indicados es el más utilizado para conocer los caudales de riego disponibles en la parcela a irrigar, mide con excelente aproximación los gastos pequeños (menos de un litro por segundo) o grandes (hasta diecisiete litros por segundo). (Briones, 2008). El error de medición del dispositivo está entre $5 \%$ y $3 \%$, lo primero corresponde si la canaleta trabaja ahogada y lo segundo bajo una descarga libre (Reddy et al., 2013).

La canaleta Parshall permite obtener medidas exactas del caudal, con una única lectura de la regleta del dispositivo, el flujo es libre y la velocidad del agua al ingreso de la garganta es mayor que la de aproximación, lo que evita la sedimentación (Xiao et al., 2016). El aforador Parshall está compuesto por tres secciones: entrada, garganta o sección estrangulada y salida, la primera presenta dos paneles verticales simétricos y convergentes, y de un fondo que es horizontal, la segunda también tiene dos paneles verticales pero paralelos, y la base es inclinada hacia abajo, la tercera consta de dos paneles verticales que divergen entre sí (Ferro, 2016).

El aforador Sin Cuello (Cutthroat Flume) está compuesto por la sección de entrada, sección de salida, garganta y la plantilla. La unión entre la entrada y la salida forma una constricción en la estructura conocida como "garganta" y el fondo es plano (Gogus et al., 2013). El medidor de caudal denominado Garganta Larga es utilizado en la medida del flujo de agua en canales abiertos, por su forma trapezoidal es de fácil adaptación a los canales de riego. Presenta una constricción entre la entrada y la salida lo que constituye la garganta y permite establecer características de flujo en régimen crítico (Azimi, 2016).Esta investigación se centra en el estudio de la medición del caudal como un componente fundamental a la hora de recolectar, distribuir, entregar y aplicar el recurso hídrico. Uno de los sistemas para esta medición en canales o acequias más ampliamente utilizado son los dispositivos portátiles por donde todo el caudal que circula pasa a través de la constricción del aforador produciéndose un cambio del régimen del flujo y mediante medidas de la altura del nivel de agua se determina el gasto (Gupta et al., 2017).

\section{Materiales y métodos}

\section{Fase de campo}

ocalización del área de estudio o lugar de medición del caudal. El sitio elegido fue un trayecto recto del canal, sin derivaciones de agua cercanas y lo suficientemente apartado hacia aguas abajo de cualquier tipo de estructura de descarga o control (ni compuertas, ni partidores) que pueda provocar alteración del flujo.

Instalación de los dispositivos portátiles.- El aforador fue ubicado en el canal de tal modo que se evitaron zonas de perturbaciones y de turbulencia. El medidor de flujo o gasto fue enterrado hasta que su fondo coincidió con la base del canal de tierra y su regla calibrada concuerde con la entrada del líquido. La nivelación se la realizó con la ayuda de un nivel automático que permitió establecer la posición horizontal tanto a lo largo como a lo ancho del tramo en estudio. Finalmente se cubrió con arcilla los bordes de entrada para evitar fugas que invaliden la medición y direccionen a todo el flujo de agua del canal a circular por el instrumento de aforo, (Anexo 1 y 2). Medición del caudal.- Antes de dar lectura del nivel de agua se esperó varios minutos hasta que el flujo se estabilizó. Se realizó nueve mediciones por cada tipo de aforador en tres puntos estratégicos, a lo largo del canal que se localizaron a una distancia de cuatro metros entre aparato y punto de derivación, siguiendo un orden de ubicación entre aforadores como la canaleta Parshall (CP), el aforador con cuello (AC) y el aforador sin cuello (AS), finalmente la ubicación de los dispositivos fue: CP-AC-AS, AC-CP-AS y AS-AC-CP con un total de cincuenta y cuatro datos que consideraron dos rangos de caudales $(0 \mathrm{~L} / \mathrm{s}$ a $2 \mathrm{~L} / \mathrm{s} ; \mathrm{y}, 2 \mathrm{~L} / \mathrm{s}$ a $20 \mathrm{~L} / \mathrm{s})$. Para identificar con mayor facilidad los dispositivos se etiquetó a cada uno de ellos. El tiempo que transcurrió entre lecturas fue de quince minutos entre puntos. Además, en la medición de los caudales se utilizo la velocidad del flujo a través del método del flotador descrito por Martínez y Castaño (2005) y el área del aforador.

\section{Fase de laboratorio}

Medición del caudal en el banco hidráulico del laboratorio.El caudal que se hizo circular en el banco hidráulico se midió con el vertedero triangular. Para medir dicho gasto fue necesario primeramente permitir que el tirante se estabilice. 
A continuación se determinó la altura total hasta la cresta del vertedero que fue de $130 \mathrm{~mm}$, luego se movió aguas arriba el medidor de tirantes, a una distancia igual a $2 / 3$ de la altura total, y se determinó la diferencia con el tirante de agua (Torres et al., 2016).

La variación entre el tirante de agua y la cresta del vertedero corresponde a la carga sobre el vertedero $(\mathrm{H})$. En la realización de cálculos se utilizó la ecuación 1, la misma que fue tomada de Çengel y Cimbala 2010.

$$
\dot{V}=C_{d v, \operatorname{tri}} \frac{8}{15} \tan \left(\frac{\theta}{2}\right) \sqrt{2 g} H^{5 / 2}
$$

En la razón de flujo para un vertedero triangular de pared delgada el rango típico de valores de $\mathrm{C}_{\mathrm{d} \text {,tri }}$ está entre $0.58 \mathrm{y}$ 0.62. Por lo tanto, la fricción del fluido, la constricción del área del flujo, y otros efectos disipativos originan que la razón de flujo a través del vertedero triangular real disminuya 40 por ciento comparado con el caso ideal. Para casos más prácticos $\left[(\mathrm{H}>0.2 \mathrm{~m})\right.$ y $\left.\left(45^{\circ}<\theta<120^{\circ}\right)\right]$. Para la aplicación de la ecuación 1 que corresponde a un vertedero triangular, el caudal está en $\mathrm{m} 3 / \mathrm{s}$, H, es la carga sobre la cresta del vertedero en $\mathrm{m}$ (Soler et al., 2011).

Medición del caudal en el banco hidráulico.- Antes de medir el caudal en el banco hidráulico se espera algunos minutos para que se estabilice el flujo. A continuación se determinó el volumen de agua en un determinado tiempo, se repitió el proceso varias veces, y se obtuvo un valor promedio del volumen. Para realizar los cálculos del caudal se aplicó la ecuación (2), recopilada de Kundu y Cohen (2002). De donde, tes el tiempo, $x$ e y son coordenadas cartesianas; $u$ componente de la velocidad de la corriente integrada verticalmente en la dirección (x); $\mathrm{v}$ es el componente de la velocidad de la corriente integrada verticalmente en la dirección (y), h es la profundidad:

$$
\frac{\partial h}{\partial t}+\frac{\partial}{\partial x}(u h)+\frac{\partial}{\partial y}(v h)=0
$$

Instalación de los dispositivos en el laboratorio.- Con la información de los procesos anteriores se comprobó que el gasto que circula, a través del canal del banco hidráulico, es constante. Al no existir variación en los resultados anteriores se asume que no existen fugas o pérdidas en el trayecto del canal.

Para la instalación de los dispositivos en el banco hidráulico se utilizó extensiones fabricadas en tol galvanizado de dos metros de longitud, que se adaptaron a los instrumentos de medición y contaron con disipadores de energía que permitieron estabilizar el tirante de agua antes de realizar las lecturas. El orden de instalación de los dispositivos fue CP, AC y AS. Se realizaron seis mediciones por cada aforador y con diferentes descargas que fueron controladas en el banco hidráulico. La primera medida fue tomada después de que el gasto se estabilizó y a cero porciento de pendiente. La segunda medida se realizó con una modificación de $1 \mathrm{~cm}$ de desnivel de la canaleta y $2 \mathrm{~cm}$ en la tercera medida, las variaciones en los desniveles corresponden a las pendientes de $0.64 \%$ y $1.27 \%$, respectivamente. Para el cálculo de la pendiente del dispositivo, se lo realizó con el uso de la ecuación 3, presentada por Çengel y Cimbala, (2010).

$$
S_{0}=\tan \alpha=\frac{z_{1}-z_{2}}{x_{2}-x_{1}} \cong \frac{z_{1}-z_{2}}{L}
$$

En la ecuación 3, $\alpha$ es el ángulo del fondo del canal respecto a la horizontal. En general, la pendiente del fondo So es muy pequeña, y por lo tanto el fondo del canal es casi horizontal. Por lo tanto, $\mathrm{L} \sim \mathrm{x}_{2}-\mathrm{x}_{1}$, donde $\mathrm{x}$ es la distancia en la dirección horizontal.

El efecto de la pendiente en el error $\mathrm{K}$ de las curvas se estimó con base en el ratio entre el caudal calibrado en el aforador al cero por ciento de pendiente y el caudal leído en el aforador instalado con cada pendiente.

También, la profundidad del flujo y medida en la dirección vertical puede considerarse como la profundidad normal del canal con un error despreciable. Si el fondo del canal es recto y la pendiente del fondo es constante, la caída vertical entre las secciones 1 y 2 puede expresarse como $z_{1}-z_{2}=S_{0} L$. Después de determinar el gasto a diferentes pendientes con el dispositivo $\mathrm{CP}$ se procedió a repetir el proceso con $\mathrm{AC}$ y AS consecutivamente.

\section{Cálculos}

Fase de campo.- Para el dispositivo CP existe una curva de calibración del fabricante que permitió determinar su descarga en función de su altura, mientras que para $\mathrm{AC}$ y AS se determinó el gasto a través de la ecuación de continuidad (2). Fase de laboratorio.- Con las mediciones realizadas en laboratorio se elaboró las curvas para los dispositivos $\mathrm{CP}, \mathrm{AC}$ y AS. Finalmente se verificó la curva del dispositivo A con la existente.

\section{Simulación}

La simulación de las canaletas se la realizó en AnsysCFD ${ }^{\circledR}$ Academic R18.0 con el fin de comparar los datos obtenidos en campo y en laboratorio.

La plataforma AnsysCFD incluye software y componentes para diferentes fenómenos físicos. Para el estudio del comportamiento de los fluídos dentro de ANSYS se despliega Fluent, un programa CFD o de análisis de fluidos permite la simulación de flujos que incluye turbulencia, reacciones o efectos térmicos. Dentro de los datos para simular el comportamiento del agua en los aforadores se emplea la altura del aforador, la densidad del agua y el volumen del agua. ANSYS FLUENT ha adoptado el enfoque EulerianEulerian para modelar el flujo. En el flujo se modela usando las ecuaciones compresibles de Navier-Stokes, además, de 
dos ecuaciones de transporte para la fracción de masa en fase líquida y el número de gotas líquidas por unidad de volumen. Los métodos numéricos son el solucionador basado en presión que emplea un algoritmo, que pertenece a una clase general de métodos llamados el método de proyección que son el algoritmo segregado basado en la presión, el algoritmo acoplado a presión, además, otro método numérico es el Solver basado en densidad, que resuelve las ecuaciones de continuidad, impulso y (cuando corresponda)transporte de energía y especie simultáneamente (es decir, acoplados entre sí). El modelo de turbulencia es k-epsilon (Ansys, 2011).

\section{Resultados y discusión}

os resultados obtenidos muestran que no existen diferencias significativas al medir los caudales de $0 \mathrm{~L} / \mathrm{s}$ a $2 \mathrm{~L} / \mathrm{s}$ y $2 \mathrm{~L} / \mathrm{s}$ a $10 \mathrm{~L} / \mathrm{s}$ de los canales con los dispositivos $\mathrm{CP}$, AC y AS (tablas 1 y 2).

A valores de caudal bajo los aforadores portátiles trabajan eficazmente y la altura del agua a la entrada del dispostivo depende de la magnitud y variación del flujo de agua ( $\mathrm{Hu}$ et al., 2014).

Tabla 1. Mediciones de gasto de $0.25 \mathrm{~L} / \mathrm{s}+/-0.01 \mathrm{~L} / \mathrm{s}$ con los tres dispositivos

\begin{tabular}{lrrrrr}
\hline \multicolumn{2}{c}{ Canaleta Parshal } & \multicolumn{2}{c}{ Aforador con Cuello } & \multicolumn{2}{c}{ Aforador sin cuello } \\
\hline Altura (mm) & Caudal (1/s) & Altura (mm) & Caudal (l/s) & Altura (mm) & Caudal (l/s) \\
\hline 19.8 & 0.25 & 50.0 & 0.25 & 14.9 & 0.24 \\
19.9 & 0.25 & 50.1 & 0.25 & 14.9 & 0.25 \\
19.9 & 0.25 & 50.2 & 0.25 & 15.0 & 0.25 \\
19.9 & 0.25 & 50.2 & 0.25 & 15.0 & 0.25 \\
20.0 & 0.25 & 50.3 & 0.25 & 15.0 & 0.25 \\
20.0 & 0.26 & 50.3 & 0.25 & 15.0 & 0.25 \\
20.0 & 0.26 & 50.5 & 0.26 & 15.0 & 0.26 \\
20.1 & 0.26 & 51.0 & 0.26 & 15.1 & 0.26 \\
20.2 & 0.26 & & $\mathbf{0 . 2 5}$ & & $\mathbf{0 . 2 5}$ \\
Media & $\mathbf{0 . 2 5}$ & & $\mathbf{0 . 0 0 2}$ & & $\mathbf{0 . 0 0 5}$ \\
Desviación Estándar & $\mathbf{0 . 0 0 3}$ & & & & \\
\hline
\end{tabular}

Tabla 2. Representación gráfica de los valores de caudal entre $5.40 \mathrm{~L} / \mathrm{s}+/-0.04 \mathrm{~L} / \mathrm{s}$ medidos con las tres canaletas

\begin{tabular}{lrrrrr}
\hline \multicolumn{2}{c}{ Canaleta Parshal } & \multicolumn{2}{c}{ Aforador con Cuello } & \multicolumn{2}{c}{ Aforador sin cuello } \\
\hline Altura (mm) & Caudal (1/s) & Altura (mm) & Caudal (l/s) & Altura (mm) & Caudal (l/s) \\
\hline 139.8 & 5.36 & 159.2 & 5.40 & 125.5 & 5.42 \\
139.9 & 5.36 & 159.5 & 5.41 & 125.5 & 5.42 \\
140.0 & 5.36 & 159.8 & 5.42 & 125.5 & 5.42 \\
140.0 & 5.37 & 159.9 & 5.43 & 125.7 & 5.43 \\
140.1 & 5.37 & 160.1 & 5.44 & 125.9 & 5.44 \\
140.1 & 5.37 & 160.2 & 5.45 & 126.0 & 5.44 \\
140.2 & 5.40 & 160.3 & 5.47 & 126.0 & 5.44 \\
140.2 & 5.40 & 160.5 & 5.47 & 126.3 & 5.45 \\
141.0 & 5.43 & 160.8 & 5.50 & 126.4 & 5.46 \\
Media & $\mathbf{5 . 3 8}$ & & $\mathbf{5 . 4 4}$ & & $\mathbf{5 . 4 4}$ \\
Desviación Estándar & $\mathbf{0 . 0 2 5}$ & & $\mathbf{0 . 0 3 1}$ & & $\mathbf{0 . 0 1 4}$ \\
\hline
\end{tabular}

Los valores resultantes de medir el gasto entre los tres dispositivos de aforo presentaron variación para diferentes caudales: Para el rango de 5.40 1/s +/- 0.04 1/s fue de $0.06 \mathrm{~L} / \mathrm{s}$ entre el dispositivo CP-AC y CP-AS, y de $0 \mathrm{~L} / \mathrm{s}$ entre AC-AS. Para descargas comprendidas entre $0.25 \mathrm{~L} / \mathrm{s}+/-0.01 \mathrm{~L} / \mathrm{s}$ no existio variación entre los dispositivos.

Mediciones de gasto. Una de las ventajas de los dispositivos estudiados es la precisión en la medicion de descargas débiles ya que otros dispositivos no la tienen (Tavares, 2005).

Curvas de calibración de los aforadores. Las curvas de calibración (figuras 1, 2 y 3) permiten obtener directamente el caudal en litros por segundo al ingresar los datos de profundidad de agua medidos con la regleta del dispositivo portátil (en milimetros). La medición del gasto es muy práctica y rápida, además la precisión de las medidas depende directamente de la nivelación correcta de los instrumentos, lo que confirma Pedroza (2012) en su estudio realizado en función del análisis de Standard Test Method for Open Channel Flow Measurement of Water with Parshall Flume de la American National Standard. 


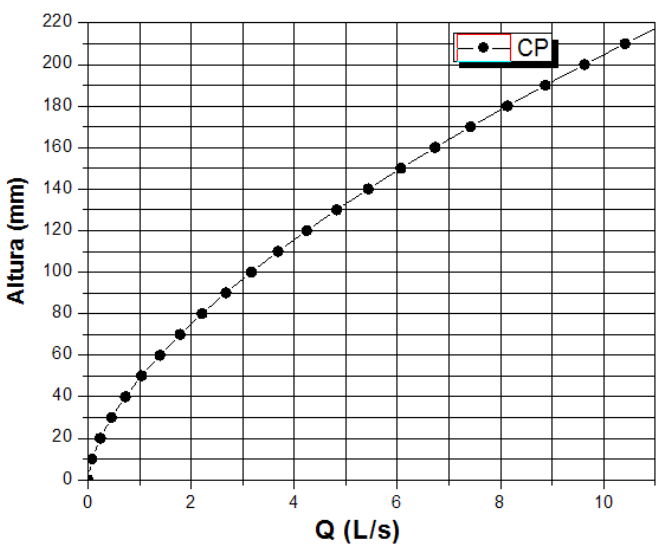

Figura 1. Curva de calibración CP

La superficie de la sección transversal de la corriente, como su velocidad, varían con la altura de agua, por lo cual, una vez conocida esa relación, pueden obtenerse los caudales por medio de las alturas de agua registradas, en escalas colocadas en forma apropiada, en las curvas de calibración de los dispositivos de aforo (Basán, 2008).

La calibración de la canaleta Parshall está relacionada con la pendiente del canal (Pedroza, 2012).

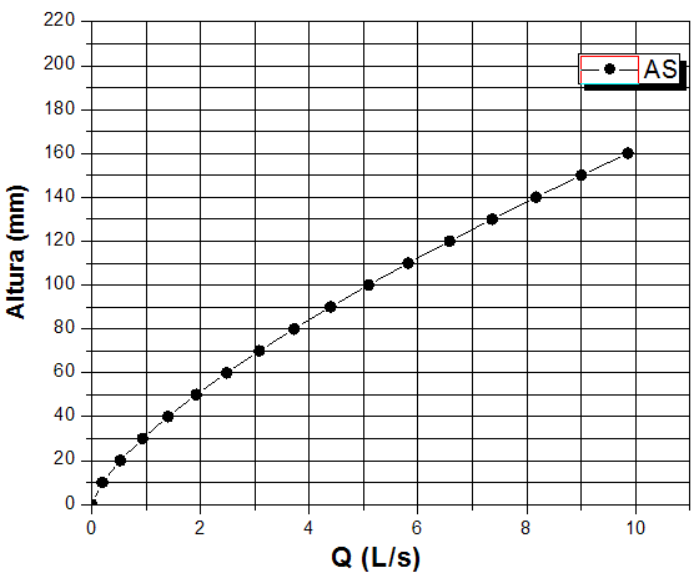

Figura 2. Curva de calibración AS

La curva de calibración de los dispositivos CP, AC y AS es necesaria para la implementación en campo de los medidores de gasto. Las curvas representan un gráfico para la medida de la descarga en 1 / s frente a la cabeza en mm para el flujo libre. La flexibilidad de los dispositivos y su fácil calibración los convierten en la mejor estructura para la mayoría de las aplicaciones de medición de caudal en canales abiertos (Das et al., 2015).

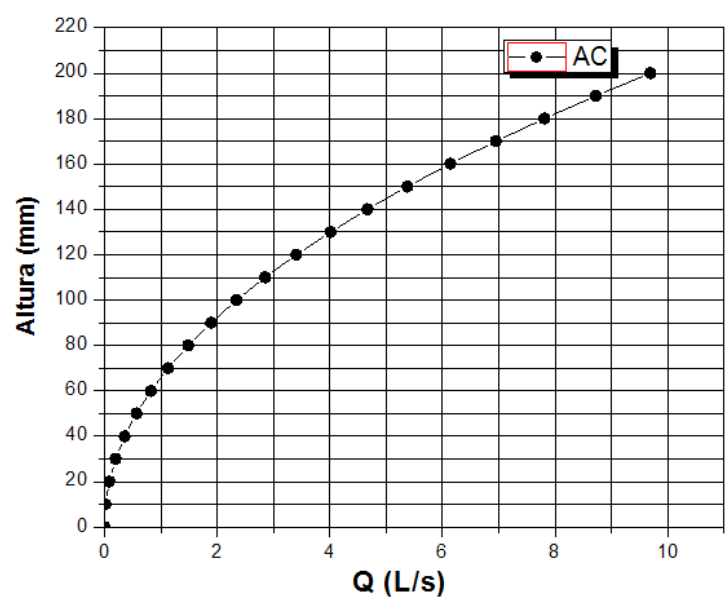

Figura 3. Curva de calibración AC

Las curvas de calibración obtenidas en laboratorio facilitan la determinación de caudales en campo.

Los resultados de los múltiples aforos practicados con los tres tipos de canaletas así como las modelaciones efectuadas, a través de dinámica de fluidos computacional de las secciones previas soportan la validación de las tres aproximaciones gráficas que se presentan como curvas de calibración para la medición del flujo en cada dispositivo reveladas en esta sección; así se presentan las figuras 1, 2 y 3 para la canaleta Parshal, aforador con cuello y aforador sin cuello, respectivamente; las mismas que de manera muy práctica permiten ser utilizadas mediante la profundiad de agua al ingreso del aforador como información de entrada, consecuentemente, el caudal será la información de salida.

\section{Ecuaciones de los dispositivos de aforo}

Las ecuaciones determinadas en laboratorio para los diferentes dispositivos de aforo funcionan correctamente cuando los instrumentos de aforo son nivelados, si existe variación de pendiente se realiza una corrección al gasto obtenido a través del coeficiente de corrección $\mathrm{K}$ y según el valor de la pendiente. Además, se debe considerar que el desnivel incide directamente en la altura del tirante y en el error de las mediciones. Ver Tabla 3. 
Tabla 3. Ecuaciones y factor de corrección (K) para los dispositivos de aforo.

\begin{tabular}{lllll}
\hline Dispositivo & \multicolumn{1}{c}{ Ecuación $(\mathbf{m}=\mathbf{0 \%})$} & \multicolumn{1}{c}{ Factor de corrección $(\mathbf{K})$} & \multicolumn{1}{c}{ Ecuación más K } \\
\hline CP & $\mathrm{Q}=0,00196 \times(\mathrm{H})^{\wedge} 1.60416$ & $\mathrm{~m}=0,64 \mathrm{~K}=1,016$ & $\mathrm{~m}=1,27 \mathrm{~K}=1,024$ & $\mathrm{Q}=\mathrm{K}^{*} 0,00196 \times(\mathrm{H})^{\wedge} 1.60416$ \\
& & $\mathrm{E}=1,5$ & $\mathrm{E}=2,1$ & \\
$\mathrm{AC}$ & $\mathrm{Q}=0,00019 \times(\mathrm{H})^{\wedge} 2.04578$ & $\mathrm{~m}=0,65 \mathrm{~K}=1,021$ & $\mathrm{~m}=1,31 \mathrm{~K}=1,051$ & $\mathrm{Q}=\mathrm{K}^{*} 0,00019 \times(\mathrm{H})^{\wedge} 2.04578$ \\
& & $\mathrm{E}=1,4$ & $\mathrm{E}=3,2$ \\
$\mathrm{AS}$ & $\mathrm{Q}=0,00787 \times(\mathrm{H})^{\wedge} 1.40541$ & $\mathrm{~m}=0,64 \mathrm{~K}=1,001$ & $\mathrm{~m}=1,28 \mathrm{~K}=1,069$ & $\mathrm{Q}=\mathrm{K}^{*} 0,00787 \times(\mathrm{H})^{\wedge} 1.40541$ \\
& & $\mathrm{E}=1,1$ & $\mathrm{E}=1,5$ & \\
\hline
\end{tabular}

$\mathrm{m}=$ pendiente en porcentaje

$\mathrm{K}=$ factor de corrección

$\mathrm{E}=$ error

$\mathrm{Al}$ incrementar la pendiente en los dispositivos de aforo, el tirante disminuye y la medida de gasto se ve afectada. Para simplificar los cálculos de gasto en los dispositivos es importante contar con la curva de calibración y un factor de corrección (Thornton, 2009).

La velocidad del agua se incrementa a la salida de la canaleta por la contracción que se encuentra en el centro de la misma. Las velocidades obtenidas en la simulación al ingreso del flujo del canal se encuentran en un rango de $0.7 \mathrm{~m} / \mathrm{s}$ a $2 \mathrm{~m} / \mathrm{s}$ lo que coincide con los datos obtenidos en campo y laboratorio. La descarga obtenida puede variar a medida que transcurre el tiempo y en el mismo día según la dotación del agua para la parcela y época del año (Fossati et al., 2014).

Las características físicas de diseño de los medidores de descarga permitieron pasar el sedimento y produjeron una superficie de agua estable y legible en la estación de medición o regleta. Las simulaciones realizadas en AnsysCFD ${ }^{\circledR}$ Academic R18.0 comprueban las condiciones presentadas tanto en campo como en laboratorio.

En la investigación actual se introduce un factor de corrección $(\mathrm{K})$ en función de la pendiente que permite utilizar los dispositivos con alta precisión para diferentes secciones de control (Vatankhah, 2012).
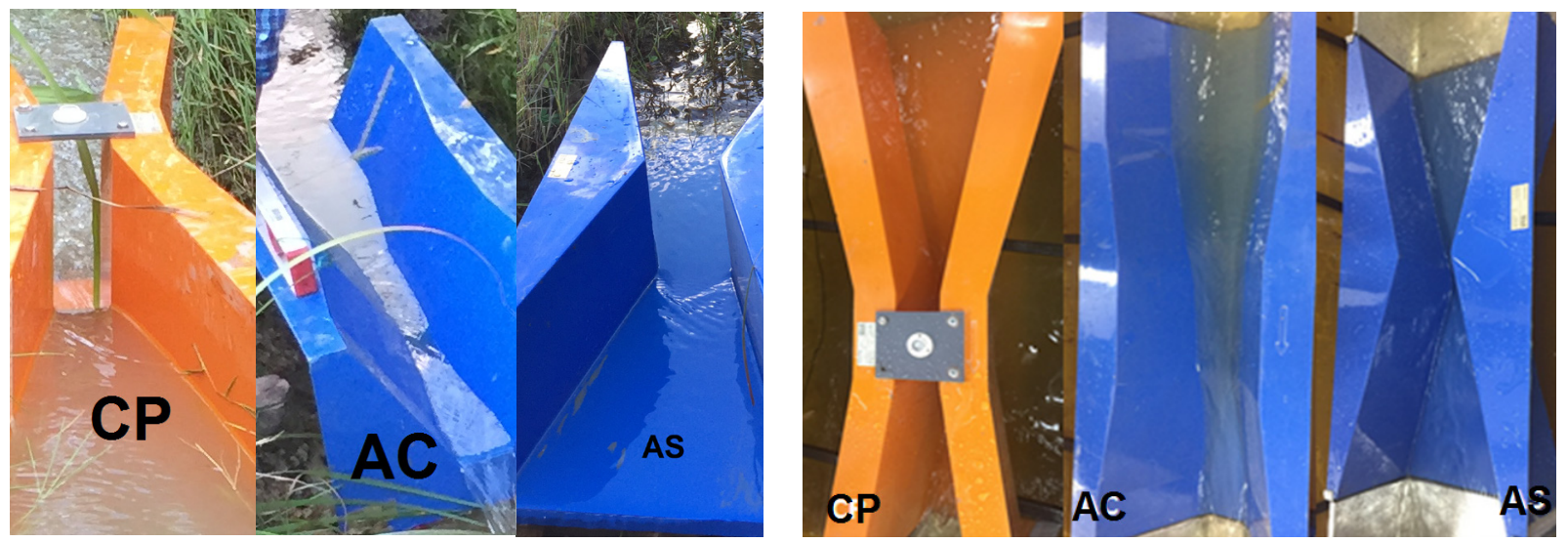

Figura 4. Fotografías de las canaletas utilizadas para los aforos en campo (izquirda) y Laboratorio (derecha).
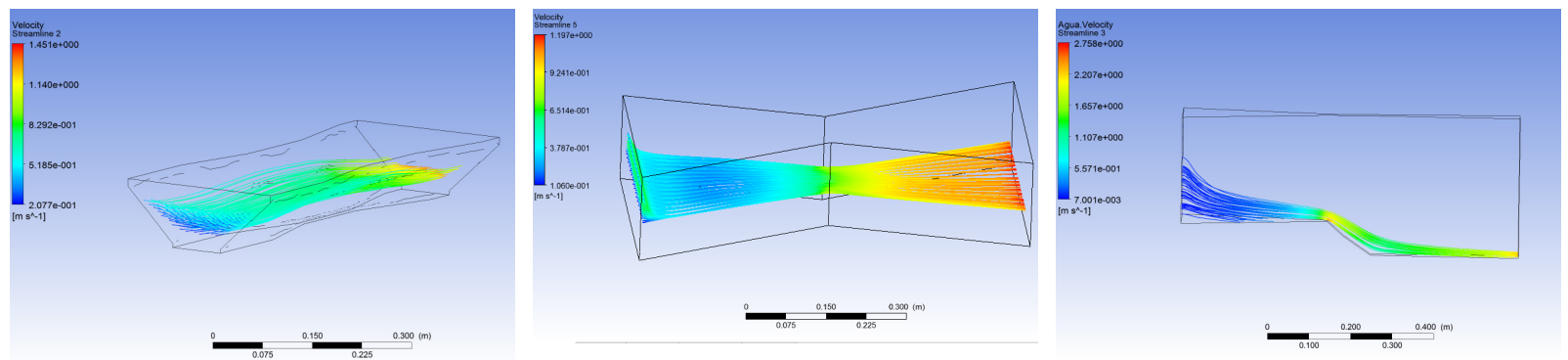

Figura 5. Gráfico con los resultados de la simulación CFD de los vectores de velocidad al interior de las canaletas. Valores de gasto $0 \mathrm{~L} / \mathrm{s}-2 \mathrm{~L} / \mathrm{s}$. 
Según los resultados de la modelación matemática (AnsysCFD) el valor de presión que el flujo ejerce en la canaleta se encuentra en un rango comprendido entre $1 \mathrm{~Pa}$ a $2 \mathrm{~Pa}$.

La mayor presión que ejerce el flujo de agua se encuentra en el fondo de la canaleta y se encuentra en un rango entre -1.64 $\mathrm{Pa}-1.65 \mathrm{~Pa}$.

En el resultado de los análisis de dinámica de fluidos computacional de las canaletas se evidencia que en la sección de la estrangulación o garganta ocurre un flujo crítico seguido de un flujo supercrítico en una corta distancia y un salto hidráulico inmediatamente aguas abajo en la canaleta Parshall, tal como lo propone Samani (2017).

La canaleta Parshall es más precisa que los otros dos instrumentos de aforo, dado que se puede eliminar la pendiente del canal (Vatankhah, 2015).
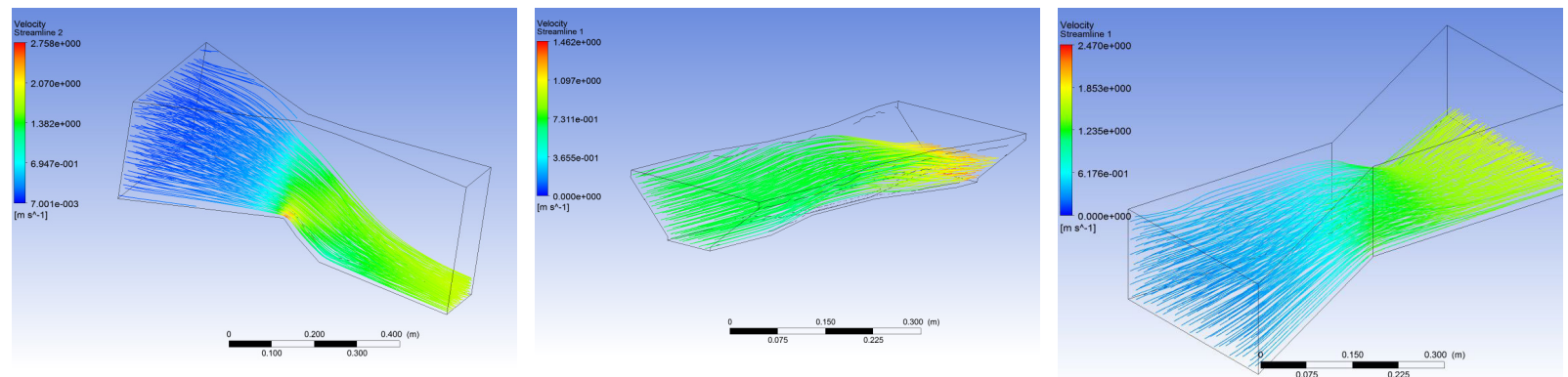

Figura 6. Gráfico con los resultados de la simulación CFD de los vectores de velocidad al interior de las canaletas. Valores de gasto $2 \mathrm{~L} / \mathrm{s}-10 \mathrm{~L} / \mathrm{s}$.
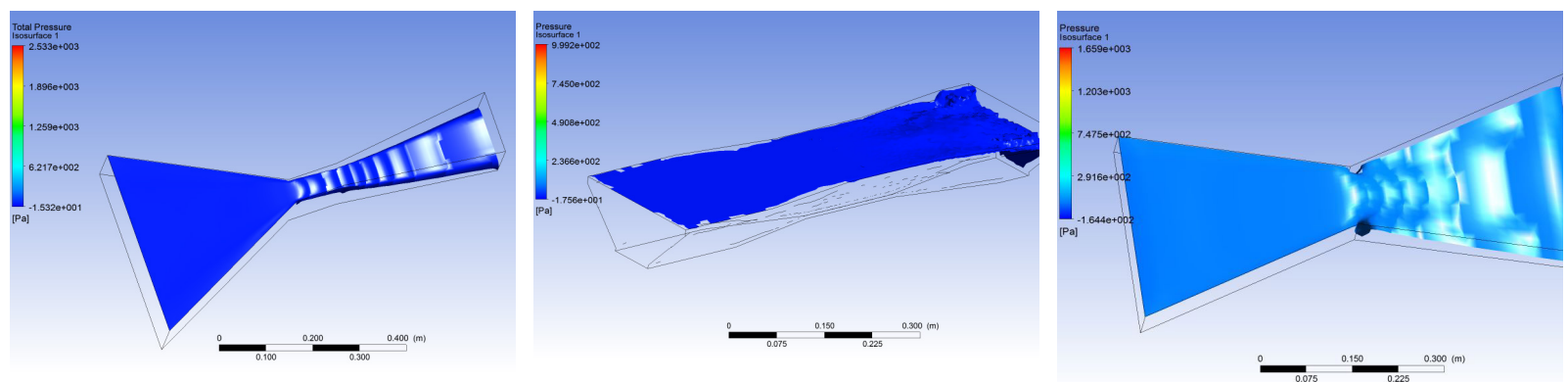

Figura 7. Resultado gráfico de la simulación de la presión en el fondo de las canaletas.

\section{Conclusiones}

$\mathrm{E}^{-1}$ n el análisis de los tres elementos típicos de aforo en pequeños canales y acequias para riego se determinó el coeficiente de corrección $\mathrm{K}$ que se encuentra relacionado directamente con el desnivel y las características del dispositivo. A mayor pendiente el error se incrementa. Por cada $1 \%$ de aumento de pendiente del aforador el error en el dispositivo CP se incrementa en un $0.95 \%$, para el AC el error es de $2.72 \%$, mientras que para el AS es de $0.62 \%$.

En el comportamiento hidrodínamico de los dispositivos se concluye que el flujo turbulento que ingresa al aforador ocasiona alteraciones en la determinación del caudal.

\section{Agradecimiento}

A 1 coordinador del Laboratorio de Hidráulica del ADepartamento Geología y Minas e Ingeniería Civil
- UTPL, Ing. Jorge Veintimilla por el préstamo de los dispositivos.

A los dueños de las parcelas agrícolas que permitieron realizar los aforos en sus propiedades.

\section{Bibliografía}

Ansys (2011). Ansys Fluent Theory Guide.

Azimi, A. (2016). Free surface flow characteristics of multiphase viscoplastic fluids on inclined flumes and planes. International Journal of Multiphase Flow 78 (2016) 5969

Basán, M. (2008). Aforadores de corrientes de agua. INTAEEA Santiago del Estero

Briones, G. (2008). Aforo Agua en canales y tuberías. Editorial Trillas, México.

Çengel, Y y Cimbala, J. (2010). Mecánica de Fluídos. Fundamentos y aplicaciones. McGrawHill. México.

Das, R., Nayek, M., Dutta, P., Mazumdar, A. (2015). Design 


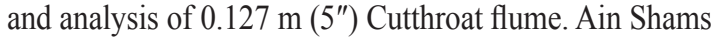
Engineering Journal.

Fossati, M., Santoro, P., Mosquera, C., Martínez, F., Ghiardo, P., Ezzatti, F., Pedocchi, I., Piedra., I. (2014). Dinámica de flujo, del campo salino y de los sedimentos finos en el Río de la Plata. Flow Measurement and Instrumentation 1(2014), 48-63

Ferro, V. (2016). Simple flume with a central bafle. Flow Measurement and Instrumentation 52 (2016) 53-56

Gogus, M., Al-Khatib, I., Atalay, A. (2013). Effect of the downstream transition region of a flow measurement flume of rectangular compound cross section on flow properties. Flow Measurement and Instrumentation 33(2013)88-95.

Gupta, S. (2017). Section: Free surface flow measurements. Flow Measurement and Instrumentation 54 (2017) 273

Hu, H., Huang, J., Qian, Z., Huai, W., Yu, G. (2014). Hydraulic analysis of parabolic flume for flow measurement. Flow Measurement and Instrumentation 37(2014), 54-64

Kundu, P., y Cohen, I. (2002). Fluids Mechanics. USA: Academic Press. http://librosysolucionarios.net/ mecanica-de-fluidos-5ta-edicion-ira-m-cohen-pijush-kkundu-david-r-dowling/

Martínez, P., y Castaño, S. (2005). Fundamentos de Hidrogeología. México: Ediciones Mundi - Prensa.

Pedroza, E. (2012). Variaciones en la calibración de un Aforador Parshall. Ciencias del Agua, III.

Reddy, M., Jumaboev, K., Matyakubov, B., Eshmuratov, D. (2013). Evaluation of furrow irrigation practices in Fergana Valley of Uzbekistan. Agricultural Water Management 117 (2013) 133- 144

Riera, C y Pereira, S. (2012). Entre el riesgo climático y las transformaciones productivas: la agricultura bajo riego como forma de adaptación en Río Segundo, Córdoba, Argentina 4611 (2012) 52-65

Samani, Z. (2017). Three Simple Flumes for Flow Measurement in Open Channels. Journal of Irrigation and Drainage Engineering, 143(6), 04017010.

Soler, J., Gómez, M., Rodellar, J. (2011). Un algoritmo para el diseño de un controlador en lazo abierto en canales 27(2011), 304-318

Tavares, C. (2005). Hidrología y variación topográfica de un riachuelo subantártico en la ensenada Mackellar, Isla Rey Jorge, Antártida. Perú: Espacio y Desarrollo N 17.

Thornton, C. (2009) Supercritical Flow Measurement Using a Small Parshall Flume. Journal of irrigation and drainage engineering, I, 90-102. doi: 10.1061/ ASCEIR.1943-4774.0000014

Torres, F., Padilla, J., Rodríguez, C., Ramírez, H,. Cantero, R.. (2016). La modelación hidrodinámica para la gestión hídrica del embalse del Guájaro, Colombia. Revista Internacional de Métodos Numéricos para Cálculo y Diseño en Ingeniería 32 (2016) 163- 172

Vatankhah, A y Mahdavi, A. (2012). Simplified procedure for design of long-throated flumes and weirs. Flow Measurement and Instrumentation 26 (2012) 79-84

Vatankhah, A. (2015). Discussion of "Supercritical Flow Measurement Using a Large Parshall Flume" by Amanda L. Cox, Christopher I. Thornton, and Steven R. Abt. J. Irrig. Drain Eng., 2015, 141(3): -1-1

Xiao, Y., Wang, W., Hu, X., Zhou, Y. (2016). Experimental and numerical research on portable short-throat flume in the field 47 (2016) 54- 61 